\title{
Alimentar a otros para alimentar a los propios: dualidades y desigualdades de la venta del trabajo de alimentar en la Ciudad de México
}

\section{Feeding Others to Feed Your Own: Dualities and Inequalities of Selling Feeding Work in Mexico City}

Recibido: 30 de julio

de 2020

Aceptado: 26 de enero de 2021

Publicado: 15 de marzo de 2021

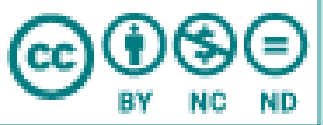

Esta obra está protegida bajo una Licencia Creative Commons Atribución-NoComercialSinDerivadas 4.0 Internacional (CC BY-NC-ND 4.0)
Paloma Villagómez Ornelas Investigadora posdoctoral, Instituto de Investigaciones Sociales, Universidad Nacional Autónoma de México (IIS-UNAM), Ciudad de México, México. Email: pvillagomez@colmex.mx, [1 https://orcid.org/oooo-0oo3-0067-5246

\section{Resumen}

El trabajo informal urbano ha sido poco estudiado en términos de las experiencias de sus actores. Este artículo analiza el caso de mujeres de estratos populares dedicadas a la venta de alimentos. El objetivo es describir la interacción entre el trabajo alimentario remunerado y no remunerado y sus efectos en la posición de género y clase de las mujeres. Mediante el análisis de entrevistas muestro que el trabajo de alimentar desarrolla dualidades subjetivas que se coproducen entre lo doméstico y lo público: en ambos espacios persiste la desvalorización del trabajo reproductivo, pero también en ambos éste puede ser fuente de orgullo $y$

CÓMO CITAR: Villagómez, Paloma. (2021). Alimentar a otros para alimentar a los propios: dualidades y desigualdades de la venta del trabajo de alimentar en la Ciudad de México. Revista Interdisciplinaria de Estudios de Género de El Colegio de México, 7, e691. doi: http://dx.doi.org/10.24201/reg.v7i1.691

http://estudiosdegenero.colmex.mx 
reconocimiento. El sentido que predomine en el balance depende de los recursos disponibles, la capacidad para movilizarlos y los rendimientos obtenidos en los negocios emprendidos.

Palabras clave: participación económica de la mujer; división sexual del trabajo; relaciones familiares.

\begin{abstract}
Studies on informal urban labor have largely overlooked the lives of the workers themselves. This article analyzes the experience of working-class women food vendors in order to describe the interaction between paid and unpaid food-related work and its effects on women's class and gender position. An analysis of interviews shows that providing food develops co-produced subjective dualities between public and domestic spheres; in both spaces, reproductive labor is disparaged but it also represents a source of pride and public recognition. The prevailing subjective balance depends on available resources, women's capacity to mobilize them, and overall business performance.
\end{abstract}

Key words: women's economic participation; sexual division of labour; family relations.

\title{
Introducción
}

A pesar de que la venta de alimentos preparados en negocios informales es parte fundamental de los sistemas alimentarios urbanos (FAO, 2007; Tinker, 1997, 2003; WIEGO, 2019), persiste una narrativa dominante que proyecta este trabajo como un problema social vinculado con situaciones de insalubridad, ilegalidad o malnutrición. Esta percepción proviene de una disputa histórica por el espacio que privilegia las formas de consumo de clases medias y altas en espacios privados, y estigmatiza las prácticas de organización y apropiación del espacio público entre sectores populares (Cross, 2000; Crossa, 2016, 2018; Hayden, 2014, 2018), lo que refuerza las desventajas del trabajo 
informal — baja productividad, remuneraciones menores e inestables, ausencia de mecanismos de protección social, subocupación - y favorece la persistencia de la pobreza.

En México 56\% de la población ocupada se dedica a actividades informales ${ }^{1}$ (INEGI, 2019a), una economía que en 2018 aportó 22\% del Producto Interno Bruto (PIB) nacional y acoge a una buena parte de los más de 52 millones de personas que viven en pobreza. En el mismo año, este sector produjo más de cinco billones de pesos, de los cuales los servicios de preparación de alimentos y bebidas aportaron 3.5\% de su valor agregado (INEGI, 2019b). Estimaciones de la organización Women in Informal Employment: Globalizing and Organizing (WIEGO), con base en la Encuesta Nacional de Ocupación y Empleo (ENOE), muestran que en 2019 alrededor de 1.5 millones de personas se dedicaban a la elaboración de alimentos y bebidas en el sector informal a nivel nacional, ya fuese desde unidades económicas de producción en los hogares, mercados o venta callejera; $70 \%$ de las personas ocupadas en esta actividad eran mujeres, de las cuales poco más de la mitad ganaban un salario mínimo o menos (WIEGO, 2019).

Desde la perspectiva del consumo, el comercio de alimentos preparados fuera del hogar absorbe cerca de la cuarta parte del gasto alimentario de una familia promedio en el país ${ }^{2}$. El peso relativo de este gasto cambia notablemente entre distintos estratos socioeconómicos, así como el significado de la práctica (Delgado y Bertran, 2010): el tercil de hogares más ricos eroga la tercera parte del gasto en el consumo de alimentos de este tipo y es más frecuente que adquieran el sentido de una ocasión especial; el tercil con menos recursos gasta la décima parte de su presupuesto alimentario en este rubro como parte de sus estrategias alimentarias cotidianas ${ }^{3}$.

En el país sabemos poco de este sector, a pesar de su importancia. La disputa económica, política y moral en torno al comercio informal y a la alimentación callejera ${ }^{4}$ obscurece las racionalidades

\footnotetext{
${ }^{1}$ De acuerdo con las definiciones más recientes, la economía informal agrupa al sector informal —micronegocios que no cuentan con los registros básicos para operar- y "otras modalidades de la informalidad", a saber, todas las variedades de trabajo que, independientemente de que estén vinculadas a actividades económicas registradas o formales, se desempeñan sin la debida protección legal como la agricultura o el servicio doméstico remunerado, entre otras (INEGI, 2019b).

${ }^{2}$ Estimaciones derivadas de la Encuesta Nacional de Ingresos y Gastos de los Hogares (ENIGH), 2018.

${ }^{3}$ Estimaciones derivadas de la Encuesta Nacional de Ingresos y Gastos de los Hogares (ENIGH), 2018.

${ }^{4}$ La Organización de las Naciones Unidas para la Agricultura y la Alimentación (FAO) entiende por comida callejera los alimentos y bebidas listos para el consumo, preparados o vendidos por personas vendedoras ambulantes o comerciantes, expendidos en la vía pública y otros lugares similares (http://www.fao.org/fcit/food-processing/streetfoods/es/). Cohen (1990) incluye los alimentos que se consumen en el lugar o los que son adquiridos para comerlos como parte del consumo familiar. Si bien estas definiciones abarcan tanto alimentos procesados como sin procesar, en
} 
y experiencias de quienes encuentran en este enclave una forma de participación económica y social, especialmente mujeres de sectores populares que parecen insertarse en él de manera casi "natural". Sabemos, sin embargo, que esta forma de participación laboral es el resultado de la construcción estructural de la diferencia sexual que asigna a las personas roles y subjetividades a partir de una idea de la división social del trabajo y del papel que el género juega en ella (Scott, 1996; West y Fenstermaker, 1995).

Este artículo explora las experiencias laborales de mujeres de estratos populares que han trabajado en la producción y venta de alimentos, ya sea en las calles o desde pequeños negocios informales instalados en sus viviendas, en la Ciudad de México. El fin es conocer cómo interactúan las experiencias remuneradas y no remuneradas del trabajo de alimentar, qué efectos tienen en la posición de género y clase de las mujeres y qué factores promueven experiencias más o menos favorecedoras, tanto en términos materiales como subjetivos. El artículo muestra que el trabajo de alimentar en el espacio público comparte con su faceta doméstica relaciones de subordinación y desvalorización del trabajo reproductivo, pero que, al igual que la alimentación familiar, esta labor puede ser resignificada en términos de satisfacción personal y prestigio frente al reconocimiento social y económico de las habilidades culinarias y la disposición al servicio. Los casos estudiados muestran que la diferencia entre ambas experiencias depende, entre otros elementos, de los acervos de recursos económicos, materiales, humanos y simbólicos al alcance.

El documento desarrolla en primer lugar la discusión planteada por los estudios de género que analizan la construcción social del trabajo de alimentar (feeding work) como parte del trabajo doméstico y de cuidado. Enseguida defino las características de la investigación de la que se desprende el análisis y presento los casos estudiados. Posteriormente, exploro en ellos tres aspectos de la venta informal de alimentos que propongo como espacios para indagar la fluidez de la posición femenina entre lo privado y lo público, y entre la organización doméstica y la social: a) las motivaciones para iniciar un negocio, b) los arreglos familiares para su desarrollo y c) las retribuciones obtenidas, sean materiales o simbólicas. En la discusión sostengo, primero, que estas tres dimensiones —no exhaustivas ni definitivas- reflejan relaciones duales de

este análisis compartimos el énfasis que hace Cohen en el trabajo implícito del proceso de transformación de los alimentos preparados, una modalidad presente en todos los casos que aquí se detallan. 
opresión/afirmación que reproducen los rasgos de la posición familiar de las mujeres en el mercado $\mathrm{y}$, segundo, que esta posición se experimenta de manera desigual conforme se tenga menor o mayor acceso a recursos económicos y sociales, lo que acentúa sus aspectos más restrictivos o constructivos.

\section{Del trabajo de alimentar}

La discusión sociológica sobre el vínculo entre la alimentación y lo familiar aparece en los estudios clásicos que Nicola Charles y Marion Kerr elaboraron a finales de la década de los años 80, en torno al papel de las prácticas alimentarias en la reproducción del orden social y las desigualdades de género y clase. Investigaciones como ésta fueron posibles gracias a la consolidación de los estudios feministas durante la década de los años $70 \mathrm{y}$ el auge de los estudios alimentarios durante los años 80 y 90. Junto con la investigación de Marjorie DeVault en torno al papel del trabajo alimentario en la reproducción de lo familiar (1991), esta serie de análisis contribuyeron a que emergieran en el campo de la alimentación formas aparentemente invisibles del trabajo doméstico femenino no pagado, analizadas como tareas que no sólo contribuyen a la subsistencia material y biológica de los hogares (Barbieri, 1978), sino también a la construcción de la idea misma de familia entendida como un dispositivo ideológico que asigna a sus miembros funciones, espacios y disposiciones afectivas y morales que reproducen la organización social con todas sus jerarquías y desigualdades de género, clase y raza (Avakian y Haber, 2005; Beagan, Chapman y Power, 2017, 2018; Charles y Kerr, 1986a, 1986b y 1986c; DeVault, 1991; Julier, 2005; Murcott, 1993).

El trabajo que realizan las mujeres para alimentar (feeding work) forma parte de una categoría más amplia de trabajos de cuidado que consisten tanto en la protección y mantenimiento de los espacios y las personas, como en la producción de sociabilidades y comportamientos que buscan apegarse a lo que se considera correcto, deseable o adecuado, no sólo en términos alimentarios, sino también de las normas del cuidado femenino y materno (Cairns y Jhonston, 2016; Bowen, Brenton y Elliot, 2019; Charles y Kerr, 1986a, 1986b; Parsons, 2015). Por medio del trabajo de alimentar, definido como planear la comida, adquirir los alimentos, prepararlos y disponerlos para el consumo, así como mantener los espacios y equipos para cocinar y comer (Mennell, Murcott y van Otterloo, 1992), se organiza la alimentación cotidiana con la generación de rutinas, estrategias, normas y 
valores que estructuran la vida familiar desde posiciones específicas de género y clase (DeVault, 1991; Parsons, 2015).

La rutinización del trabajo de alimentar y el uso que hace de formas de conocimiento aparentemente poco articulado, aportan al trabajo alimentario un sentido de espontaneidad que lo hace parecer misterioso e incluso místico, lo que facilita que esta labor se perciba como una especie de don o habilidad natural que sólo puede ser encarnada y transmitida entre mujeres (DeVault, 1991; Oleschuk, 2019). La naturalización del cuidado como un atributo o rasgo de lo femenino, y no como un trabajo que se puede elegir, negociar o resistir, favorece la reproducción de la desigualdad en la distribución del trabajo reproductivo, mediante dimensiones cognitivas y emocionales que hacen que las mujeres se sientan convocadas a servir a otros porque les parece correcto (Ehrenreich y English, 2005; Parsons, 2015; O’Connell y Brannen, 2016).

Esta orientación hacia el cuidado no se reserva para el espacio familiar, sino que forma parte del servicio pagado del trabajo alimentario. La venta informal de alimentos que realizan las mujeres retoma de la alimentación doméstica tareas, roles, relaciones sociales y hasta disposiciones afectivas que las mujeres adaptan al trabajo pagado. Este ajuste permite extender el análisis del trabajo de alimentar del espacio familiar al mercado laboral, una operación útil para aproximarse al estudio de la participación laboral femenina en sectores informales donde la posición de las mujeres podría acentuar su subordinación por al menos dos razones: primero, porque la división social del trabajo asigna menos valor a las formas de producción más vinculadas a la reproducción que a la acumulación de capital (Barbieri, 1978; Cohen, 1990; Rodríguez, 2015) y segundo porque la transacción económica que implica el trabajo alimentario pagado se monta sobre desigualdades previas de género y clase que reproducen vínculos de dominación y servidumbre (DeVault, 1991; McLean, 2013; Parsons, 2015).

La investigación más reciente sobre la intersección entre los estudios alimentarios y los de género reconoce estas relaciones y jerarquías, pero destaca la plasticidad de lo familiar y de la posición de las mujeres en los espacios públicos y privados, entendidos más como flujos de desplazamientos que como una dicotomía fija (McLean, 2013; Parsons, 2015). Abarca (2006, 2007), por ejemplo, destaca el potencial de las cocinas, especialmente las que se comparten con otras mujeres, como 
espacios para la construcción intersubjetiva de una idea de ser mujer y de cuidar, que desafía la ideología patriarcal. Otros estudios identifican la alimentación y sus labores como prácticas por medio de las cuales las mujeres impugnan las representaciones socioculturales hegemónicas de lo femenino y resignifican su trabajo como una forma de autodescubrimiento, expresión o satisfacción emocional y sensorial (Avakian y Haber, 2005; McLean, 2013).

Así, el análisis de las prácticas y los discursos culturales alrededor del trabajo alimentario expresan una tensión entre considerarle un sitio de confinamiento de lo femenino o un ámbito de agencia con efectos positivos sobre la identidad, una discusión central en los estudios feministas postcolonialistas y postestructuralistas. La diferencia entre ambas experiencias no se vive como una dicotomía tajante, sino como un continuo sobre el que las mujeres se desplazan dependiendo de la heterogeneidad de sus circunstancias de clase, etnia/raza u origen, y del acceso que tienen a recursos materiales y económicos que sostengan su participación en el trabajo alimentario, tanto doméstico como remunerado (Bowen, Brenton, Elliot, 2019; Cairns y Johnston, 2016). Así, el llamado al que me siento convocada es el de encontrar matices e identificar las tensiones y los empalmes entre uno y otro sentido del trabajo alimentario.

\section{Aproximación metodológica}

El análisis de las experiencias de las mujeres en la venta de alimentos deriva de mi investigación doctoral, cuyo objetivo fue analizar la organización de las prácticas alimentarias entre familias residentes en barrios populares (Villagómez, 2019). El estudio se llevó a cabo entre 2016 y 2019, en colonias de la alcaldía Iztapalapa, en la Ciudad de México.

La exploración de la organización de las prácticas se realizó mediante una metodología cualitativa con orientación etnográfica que incluyó la elaboración de 13 estudios de caso producidos mediante entrevistas en profundidad y observación participante en hogares. Cada caso aportó información que permitió explorar la constitución de las prácticas alimentarias familiares a partir de las siguientes dimensiones: a) la composición del hogar; b) sus condiciones económicas y materiales; c) la participación y distribución del trabajo remunerado y no remunerado; d) la organización del trabajo alimentario familiar; e) el estado de salud de la familia; f) la ocurrencia de crisis y 
dificultades en torno a la alimentación; g) la valoración de los hábitos alimentarios. Estos temas fueron tratados con la persona encargada de la alimentación en el hogar, que en todos los casos fueron mujeres.

La selección de los casos estuvo guiada por la muestra analítica diseñada para este fin ${ }^{5}$. Si bien la concentración de población en situación de pobreza en la zona es alta ${ }^{6}$, el trabajo de campo mostró que esta condición no se expresa de forma homogénea en los hogares, sino que existen variaciones en el nivel de ingreso, la calidad de las viviendas o la acumulación de activos - vehículos, maquinaria de trabajo, entre otros- que, junto con la composición sociodemográfica del hogar, producen diferencias en el nivel de bienestar que afectan la organización de las prácticas alimentarias y la experiencia subjetiva de las mismas. Por esta razón, se distinguieron niveles de precariedad alta, media y baja, definidos a partir de las variables sociodemográficas y económicas de interés ${ }^{7}$.

Las experiencias de la venta de trabajo alimentario registradas en la historia de las familias ocurrieron en los dos primeros grupos. Si bien la venta de alimentos no fue el objeto inicial de la investigación, los estudios de caso revelaron la importancia de esta actividad como una alternativa económica socorrida por las mujeres en distintos momentos de la historia familiar. El análisis de estas experiencias resultó relevante para profundizar en la trayectoria económica del hogar y comprender la posición desde donde las mujeres interpretaban su condición, a partir de su experiencia en el trabajo de alimentar, tanto dentro del hogar como fuera de él.

\section{Las mujeres y sus negocios ${ }^{8}$}

\footnotetext{
${ }^{5}$ La construcción de la muestra analítica se basó en variables sociodemográficas asociadas con el nivel de bienestar de los hogares y cuya relación con la configuración de las prácticas alimentarias interesaba conocer, a saber, la estructura del núcleo familiar, el ciclo doméstico y el sexo de la jefatura económica del hogar.

${ }^{6}$ De acuerdo con el Consejo Nacional de Evaluación de la Política de Desarrollo Social, Coneval, en 2018, 30.6\% de los habitantes de la Ciudad de México se encontraba en pobreza y $1.7 \%$ en pobreza extrema (Coneval, 2021a). En 2015, en la alcaldía de Iztapalapa estos niveles ascendían a 35.0\% y 1.7\%, respectivamente (Coneval, 2021b).

${ }^{7}$ Esta clasificación consideró las siguientes variables: ciclo doméstico, sexo de la jefatura, estructura familiar, tamaño, nivel máximo de escolaridad; y económicas: relación de dependencia económica, fuentes de ingreso, ingresos totales y per cápita, relación del ingreso respecto al costo de la canasta básica general y canasta básica alimentaria. Para revisar los perfiles agrupados en cada categoría ver Anexo 1.

${ }^{8}$ Entiendo por negocio el emprendimiento de una actividad económica de producción o comercio de bienes o servicios para su venta en el mercado, sea formal o informal. Es el equivalente a las "enterprises" a las que alude Cohen (1990).
} 
En conjunto, tenemos a siete mujeres que en distintos momentos de su vida se han dedicado a la venta de comida. Dos casos que actualmente se encuentran en una situación de menor precariedad establecieron cocinas económicas en sus viviendas, donde atendían a comensales y pedidos para llevar. Las pequeñas cocinas de Julia (72 años de edad al momento del estudio) y Rebeca (53) atendían principalmente a personal educativo y de salud de la zona, además de algunos comerciantes locales y familias vecinas. En promedio servían o repartían alrededor de 50 comidas diarias. Entre la planeación, adquisición, preparación, servicio y entrega a domicilio de los alimentos, las jornadas de trabajo eran incluso de doce horas, de lunes a viernes. Estos negocios se caracterizaron por la participación intensiva de otros miembros familiares. Estuvieron activos por ocho y nueve años, respectivamente.

Las familias con mayores restricciones económicas y materiales establecieron pequeños negocios que ofrecían alimentos menos elaborados. Las tres mujeres en esta situación — Maricela (36), Beatriz (35) e Irma (64) - vendían sándwiches, tortas o botanas, es decir, alimentos que requerían menos inversión y poco trabajo de transformación. Las condiciones materiales y espaciales de sus viviendas eran y siguen siendo de mayor carestía; la irregularidad e ineficiencia de los servicios, así como las limitaciones de espacio y equipo, impiden que las casas funcionen como activos para el trabajo. Las mujeres atendían puestos, que colocaban en la calle, durante cuatro o cinco horas a las que hay que sumar las que se invertían en el trabajo previo de compra de insumos, preparación y mantenimiento del equipo (freidoras, vitrinas, puestos metálicos) adquirido con préstamos. Los negocios iniciaron en asociación con alguna otra mujer de la familia quien terminó por abandonarlos, lo que provocó una sobrecarga de trabajo que, entre otros factores, las llevó a desistir poco tiempo después.

Finalmente, tenemos un par de mujeres que participaron en la venta de alimentos desde posiciones asalariadas. Una de ellas, Ana (28), trabajó en el sector formal como cocinera en una cadena de restaurantes. María, a sus 77 años, trabajaba como cocinera en el comedor comunitario de una de las colonias visitadas ${ }^{9}$. En ambos casos, el trabajo de estas mujeres se realizaba fuera de sus viviendas, en horarios establecidos por sus empleadores - alrededor de nueve horas- y con

\footnotetext{
${ }^{9}$ Los comedores comunitarios son parte del Programa de Comedores Populares de la Ciudad de México y no pueden ser considerados negocios, sino trabajo voluntario. Sin embargo, de acuerdo con las reglas de operación vigentes al momento de la investigación, sus trabajadores pueden recibir un salario (Gaceta Oficial de la Ciudad de México, 2019).
} 
recursos materiales provistos en sus empleos. La primera llegó a ganar 3,500 pesos quincenales y la segunda percibe 120 pesos diarios.

Aunque en diferentes circunstancias, todas estas iniciativas fueron emprendidas por mujeres que descubrieron que podían intercambiar su fuerza de trabajo, habilidades y conocimientos en un mercado que contempla espacios flexibles en los que pueden cumplir con mandatos enraizados profundamente en sus identidades como cuidadoras y cocineras (Cohen, 1990; Cohen, Bhatt y Horn, 2000). Enseguida veremos cómo los aspectos opresivos o liberadores de la venta del trabajo alimentario no se viven como experiencias dicotómicas excluyentes, sino como vivencias subjetivas complejas que se mezclan o empalman. Para analizarlo me concentro en tres dimensiones de las experiencias laborales en las que estas tensiones emergieron: las motivaciones para iniciar sus negocios, los arreglos familiares ante el trabajo y las retribuciones obtenidas. En cada aspecto distingo en las narrativas los elementos destacados como positivos de aquellos negativos que reforzaban el sentimiento constrictivo, tanto de la desigualdad de género, como de la precariedad económica.

\section{Las motivaciones para vender comida}

Antes de unirse y formar sus propias familias, algunas mujeres contaban con experiencia laboral en trabajos informales de baja calificación, generalmente mal remunerados y en ocasiones vinculados a la alimentación ${ }^{10}$. Al casarse dejaron de trabajar, a veces obligadas por sus esposos quienes las "sacaron" del mercado laboral. Sin embargo, frente a la necesidad posterior de obtener mayores recursos buscaron empleo en circunstancias más complejas, pues ya tenían hijos que cuidar y maridos con los cuales negociar o solicitar autorización. En estas circunstancias, las motivaciones de las mujeres para trabajar de manera remunerada corresponden con tres escenarios principales: 1) la existencia de crisis o necesidades extraordinarias en el hogar; 2) alteraciones en la trayectoria laboral de sus parejas; 3) la indisposición de sus cónyuges a proveer. Estos escenarios

\footnotetext{
${ }^{10}$ Julia trabajó en la cocina de un hospital, Rebeca en la de un restaurante italiano y Beatriz en una pequeña empresa de sistemas informáticos. En el ámbito informal, María fue empleada doméstica y cocinera en su juventud; Maricela trabajó en un taller de costura. Son, todas, actividades precarias con remuneraciones menores, baja calificación, mayor inestabilidad, reducida movilidad y, generalmente, sin acceso a mecanismos de protección social (Ariza, 2006).
} 
coinciden notablemente con los hallazgos de la literatura sobre trabajo femenino en contextos de pobreza (Cerrutti, 2000; González de la Rocha, 1994; Kabeer, 2010; Khurana, 2015).

En el primer escenario tenemos a Irma y Julia (precariedad alta y media, respectivamente). Recién llegadas a las colonias, sus familias vivían en habitaciones frágiles y provisionales. Lo que ganaban sus esposos - ambos trabajadores independientes de la construcción - sólo alcanzaba para la subsistencia familiar, así que ellas comenzaron a trabajar. Ambas preparaban tortas, Irma de manera asalariada y Julia por su cuenta. Junto con sus respectivas parejas decidieron que el dinero ganado por cada quien se destinaría a los gastos familiares o a la construcción. Más de 30 años después, la casa de Irma sigue en obra gris. Julia logró avanzar más, lo que después la animó a inaugurar una cocina económica en su casa. Emplearse como cocineras pareció una opción al alcance de ambas; Julia, con la secundaria terminada, tenía experiencia en grandes cocinas por su trabajo previo en un hospital y confiaba en sus habilidades. Irma, sin haber concluido la primaria y con cuatro hijos pequeños a los que no podía dejar solos demasiadas horas, salió a buscar empleo y encontró uno de medio tiempo en un establecimiento de tortas.

En el segundo escenario, relacionado con las trayectorias laborales de los cónyuges, tenemos a Beatriz, Maricela y Rebeca (con estudios de bachillerato, secundaria y primaria incompleta, respectivamente). En algún momento sus familias sufrieron eventos extraordinarios que motivaron a estas mujeres a emplearse: Carlos, pareja de Maricela, enfermó y su incapacidad produjo una severa merma en los ingresos; Alfonso, esposo de Rebeca, se quedó sin empleo como mesero y, dada su edad —más de 50 años—fue difícil encontrar otro; Humberto, marido de Beatriz, cambió de trabajo a uno de menor remuneración con tal de tener acceso a seguridad social. Después de probar soluciones como la venta de artículos por catálogo o pedir dinero prestado a familiares, las mujeres comenzaron a vender comida en puestos en la calle o, en el caso de Rebeca, en su cochera, donde montó la cocina económica que atendió durante nueve años.

Maricela y Beatriz no tenían experiencia cocinando más que para su familia. Emprendieron negocios de comida callejera porque consideraron que ésta no requería preparaciones complejas o demasiados recursos, además de que parecía una actividad redituable. También les permitía permanecer cerca de casa, lo que desde su posición como cuidadoras representaba una ventaja. Por 
el contrario, Rebeca confiaba en su experiencia previa en la cocina de un restaurante, y en la de su marido como mesero; entre los dos reunían habilidades culinarias y de servicio que, junto con la disponibilidad de una vivienda relativamente adecuada, hacían viable su negocio de comida corrida.

El tercer escenario suma a la precariedad económica la falta de compromiso o voluntad de los varones para proveer. Varias mujeres se han encontrado en esta situación, ya sea de manera intermitente o permanente. Julia señala frente a su marido:

Hubo altas y bajas. Acá (dice señalando a Martín) me respondía, a veces no me respondía. Y yo decía no, mis hijas no se van a quedar sin comer. Siempre yo he llevado la batuta en todo. Siempre yo le decía a él 'ya, o asientas cabeza o ya... Para mantenerte, mejor me mantengo yo y mis hijas'.

La pareja de Ana abandonó el hogar dejando a su cargo la manutención de la hija de ambos y el pago de una deuda de diez mil pesos. Ella comenzó a buscar trabajo y encontró una vacante como cocinera en una cadena de restaurantes; decidió solicitar el empleo porque le gusta cocinar y considera que desde pequeña lo hace muy bien. A pesar de encontrarse en una depresión profunda, trabajar por su hija fue un incentivo importante que hoy le permite presentarse como un ejemplo de esfuerzo y trabajo.

La respuesta no confrontativa de las mujeres ante la negligencia masculina consiste en arreglárselas para cubrir las necesidades familiares que queden pendientes o incompletas, una situación de subordinación que favorece la creencia de que el trabajo de las mujeres es una forma de ayuda al esfuerzo masculino, ya sea porque consideran su aportación de poco valor o por la creencia de que sus contribuciones no les otorgan el mismo nivel de autoridad (García y Oliveira, 2006; Khurana, 2015). Esta percepción se ve reforzada por el hecho de que las actividades económicas que emprenden son similares o idénticas a las domésticas, lo que extiende su falta de valor y de reconocimiento hacia el mercado de trabajo y el espacio público (Gal, 2002). 
La socialización familiar en el trabajo de alimentar, la experiencia previa en el mercado laboral y en el servicio alimentario o el conocimiento de experiencias de pares son elementos que hacen que este tipo de actividades, consideradas "femeninas", parezcan viables y socialmente legítimas para mujeres con poca formación educativa y muchas responsabilidades de cuidado familiar, las cuales limitan sus opciones, e incluso su movilidad en el espacio doméstico y barrial. Así, las motivaciones que llevan a las mujeres a vender su fuerza de trabajo para alimentar se encuentran justo en la intersección de las desigualdades de género y de clase: su posición doméstica subordinada es interpelada por las crisis o la precariedad crónica, orillándolas a realizar lo que entienden que es su deber como madres, recurriendo a las habilidades y recursos que su propia socialización como mujeres les plantea como moralmente aceptables (Cohen, 1990). Estos recursos, sin embargo, no son inherentes a la condición femenina ni iguales entre todas las mujeres, por lo que la naturaleza y el alcance de los negocios varían.

\section{Los (des)arreglos entre el trabajo público y el privado}

Las iniciativas económicas de las mujeres requirieron distintos arreglos entre el trabajo doméstico no remunerado y el remunerado que, dependiendo de sus relaciones familiares y del soporte económico y material con el que contaran, representaron ventajas o desventajas para el desarrollo de sus actividades y para su propia posición.

Por un lado, tenemos casos de precariedad moderada como los de Julia o Rebeca. Ellas pudieron establecer sus cocinas-comedores y mantenerlos durante más de ocho años, gracias a que lograron reunir recursos para invertir en insumos y equipo, además contaban con habilidades para preparar comidas elaboradas. Asimismo, tenían viviendas propias, amplias, bien ubicadas y funcionales, adaptables a una función económica de esta naturaleza. Esto implicó abrir la intimidad doméstica a extraños, algo difícil de hacer cuando se considera que las propias condiciones de vida no son dignas o presentables. Su clientela, conformada por profesores, médicos, comerciantes o vecinas, convertía a las cocinas en espacios de interacción y confianza que se sumaban a las redes de intercambio y apoyo que las mujeres construyen para cumplir con la carga de trabajo productivo y reproductivo ${ }^{11}$.

\footnotetext{
${ }^{11}$ Tanto Julia como Rebeca cuentan que las vecinas que tenían empleos fuera de su hogar dejaban pagadas las comidas de sus hijos, quienes las recogían al salir de las escuelas o las consumían ahí, bajo el cuidado de las cocineras y sus
} 
Estos trabajos también son fuentes de empleo para otros familiares. Como señalan Tinker (1997) y Abarca (2007), conforme los pequeños negocios de comida se consolidan convocan la presencia de más familiares que contribuyen a hacerlos crecer y estabilizarse, sin que ello implique necesariamente renunciar a las jerarquías y a la división de roles. Donde existía participación familiar de otras mujeres, era común que laboraran sin pago - especialmente si eran corresidentes-, lo que facilitaba pensar en su actividad más como apoyo que como trabajo. Los varones participaban sobre todo en ventas, atención o adquisiciones, tareas más vinculadas con el aspecto público que con el trabajo privado de la preparación de la comida.

La venta de antojos callejeros entre las mujeres con menos recursos tiene una lógica distinta, más precaria. El consumo es efímero, de paso, lo que tiene implicaciones en la infraestructura de los puestos —con andamiajes temporales, prácticamente sin mobiliario y acceso inestable a electricidad, gas y agua - y en las condiciones de trabajo de las mujeres. Los pequeños puestos de Maricela y Beatriz se encontraban dentro de las colonias, más cerca de sus casas que de las vías principales. Esto limitaba su exposición a clientes, pero les daba cierta seguridad, toda vez que trabajaban durante las noches de los fines de semana. Los procesos culinarios transitaban entre la casa y la calle, haciéndolos un poco más complicados, incluso caóticos: las mujeres preparaban una parte de la comida en la casa y la otra en la vía pública, llevaban y traían instrumentos de cocina y repartían botanas a los clientes mientras alimentaban a sus propios familiares. En estas circunstancias, la división entre el trabajo alimentario familiar y para la venta se dilúá, evidenciando que, bajo ciertas circunstancias, la distinción entre espacios públicos y privados es más un flujo que una dicotomía funcional (Gal, 2002; McLean, 2013; Abarca, 2006).

Ninguno de estos negocios duró más de un año. La aparición de competencia y el descenso de las ventas, aunadas a la sobrecarga de trabajo, terminaron por desalentar a las mujeres. Beatriz sentía culpa por involucrar a sus hijos en el trabajo, así fuese en tareas sencillas, pero sobre todo por dejarlos solos en casa encerrados. Maricela sentía algo semejante respecto a su hija pequeña, cree que la expuso a muchos riesgos. Aunque en otra modalidad de trabajo, a Ana también la invadió la

familiares. Cuando no tenían dinero las madres pedían la comida fiada. En más de una ocasión los alimentos se regalaban no sólo a ellas, sino a otros vecinos que se encontraban en situaciones muy precarias. 
culpa y el temor de ser una madre parcialmente ausente en la vida de su hija, lo que provocó que renunciara a su absorbente trabajo como cocinera, a pesar de que le gustaba y obtenía ingresos suficientes y seguridad social.

Es decir, ante el peso del mandato social y la falta de apoyo, hay mujeres que terminan por sentirse ansiosas y culpables incluso cuando intentan mejorar las condiciones de vida de su familia, una tensión especialmente recurrente entre personas con menos recursos que no pueden compensar o sustituir sus cuidados con servicios pagados o en instituciones públicas (Arriagada, 2005; Oliveira, Eternod y López, 1999) y que viven en condiciones materiales limitadas e inseguras que elevan el riesgo de que sus hijos sufran algún percance.

Así, la sobrecarga de trabajo que recae sobre las mujeres, para que cumplan con sus responsabilidades domésticas y contribuyan económicamente a la manutención del hogar, las orilla a buscar opciones de trabajo que permitan ajustar sus relaciones familiares y espaciales en arreglos que demandan la continuidad estratégica de su identidad como procuradoras de cuidados $\mathrm{y}$, en este caso, como cocineras ya no sólo de sus familias sino también de extraños a los que alimentan para poder alimentar a los suyos. Desafortunadamente, parece que sólo en circunstancias de menor vulnerabilidad preexistente estas experiencias mejoran la posición de las mujeres en términos de su autoridad y de su participación en prácticas más equitativas en la organización familiar.

\section{Retribuciones del trabajo: más allá del dinero}

Aunque en el imaginario popular el trabajo informal es pensado como una salida fácil, los estudios acerca de este sector coinciden en describirlo como una estrategia de subsistencia, no como una actividad que provoque el crecimiento económico y la movilidad social (Pérez, 1999; Pérez y Mora, 2006). Si bien la venta de alimentos en pequeños negocios informales no es necesariamente la única salida de las familias (Cohen, 1990), como encontró Tinker (1997) en África y Asia, esta actividad no suele tener aspiraciones de enriquecimiento o expansión, está enfocada en pervivir.

Las ganancias de los negocios que conocimos apenas rebasan sus escasas inversiones, su aportación al conjunto de ingresos familiares contribuye a superar las líneas de pobreza por un estrecho 
margen ${ }^{12}$. Julia se considera satisfecha si de los 500 pesos que invierte en la comida que vende los fines de semana, logra sacar 200 o 300 pesos adicionales. Maricela dividía los 600 pesos que obtenía en dos noches de venta de antojitos entre dos personas (ella y su suegra, quien le prestó dinero para una freidora). Beatriz y su hermana obtenían entre 500 y 600 pesos por noche y los repartían en partes iguales. Claramente, nadie podría enriquecerse con estas ganancias.

Sin embargo, a pesar del cansancio potenciado por su combinación con el trabajo doméstico familiar, algunas mujeres consideraban que el esfuerzo valía la pena. Para Beatriz los rendimientos no eran cuantiosos, pero tenía una fuente de ingresos adicional al salario de su marido para cubrir gastos escolares, de alimentación o de vivienda. Este uso del dinero se repetía en todos los casos donde las mujeres tuviesen trayectorias laborales remuneradas y, sobre todo, donde hubiera menores de edad, un fenómeno bien documentado en la literatura sobre trabajo femenino remunerado (Carney, 2015; Cohen, 1990; Khurana, 2015; García y Oliveira, 2006).

Además del ingreso y la sobrecarga de trabajo, otros factores contribuyeron a definir el éxito o fracaso de los negocios. Algunos de ellos son externos, como la precariedad del propio mercado informal, su fragilidad ante eventos externos — crisis económicas, de salud o alimentarias- o el bajo poder adquisitivo de sus consumidores. Otros son resultado de procesos estructurales de la propia socialización de las mujeres quienes, al enfrentarse a estas actividades, notan que carecen de los conocimientos o la práctica necesaria para administrar sus comercios, negociar con proveedores, incorporar innovaciones para competir con otros actores locales, entre otras actividades propias del espacio público para el que las mujeres se descubren poco preparadas.

A los cuatro o cinco meses de haber comenzado a vender con éxito, un vecino de Beatriz se instaló en la calle, vendiendo lo mismo:

Fíjate que sí [nos iba]bien, gracias a dios... Nada más que empezó a bajar la venta porque, la gente, ya sabes cómo es. Se puso al lado de nosotros, y hasta la fecha está ahí, un chavo y empezó a vender exactamente lo mismo. Entonces, sí nos dio el

\footnotetext{
${ }^{12} \mathrm{Al}$ momento de realizar el estudio, la canasta alimentaria básica costaba $\$ 1490.86$ por persona al mes, mientras que la canasta de bienes alimentarios y no alimentarios ascendía a \$2989.13, de acuerdo con la información actualizada periódicamente por el Coneval (2021c).
} 
bajón. Sí vendíamos, pero ya no era igual. Fue cuando nos dimos cuenta de que en realidad nomás le estábamos dando vuelta al dinero (...) En realidad ya casi no veíamos ganancias.

Parte de la dificultad de las reglas del juego de la informalidad callejera es su laxitud y el hecho de que esté abierto para todos. Lo que parece una ventaja para las mujeres puede terminar volviéndose en su contra. Beatriz fue desplazada por un varón que muy posiblemente tenía menos obstáculos domésticos para desarrollar esta actividad. Como han documentado García y Oliveira (2004) y Oliveira y Ariza (2000), el desplazamiento de las mujeres trabajadoras informales por parte de varones desempleados es una situación común de los mercados de trabajo precarizados. Cohen encuentra que en la venta de comida callejera, son ellos quienes tienen mayor capacidad para adaptar innovaciones tecnológicas o abrirse a nuevos productos (1990). El hecho de que las mujeres se apeguen más a platillos y técnicas consideradas tradicionales puede ser interpretado como una preferencia "cultural" que en realidad esconde una forma de rezago derivada de la propia división sexual del trabajo.

Después de varios años de actividad ininterrumpida, las ventas en la cocina económica de Rebeca comenzaron a bajar de la mano de varias crisis externas. En 2009 la epidemia de influenza AH1N1 que azotó a la Ciudad de México, redujo sus ingresos a prácticamente la mitad (de 60 comidas servidas al día a sólo 30). Con esfuerzo lograron mejorar el nivel de ventas, pero llegó un segundo golpe en 2010 que Rebeca atribuye a una crisis económica generalizada, "[L]a gente no tenía dinero... De ahí ya no nos pudimos recuperar, a veces se vendía, a veces no". La comida comenzó a quedarse rezagada y terminaban comiéndola en casa, contribuyendo al sobrepeso de la familia. Poco después su marido falleció y a la indisposición anímica se sumó la insostenibilidad de la carga de trabajo, marcando el final definitivo del negocio.

Ya sea por pérdidas económicas o, como vimos antes, por la culpa que genera el creer que no realizan eficientemente su trabajo como madres, las mujeres terminan por abandonar las iniciativas con el sentimiento de haber fracasado y con la premura de tener que conseguir más recursos. Estas narrativas conviven, sin embargo, con otras que rescatan valores que podrían encontrarse más allá de la remuneración económica, como el esfuerzo, la iniciativa y el dominio del oficio, aspectos 
exaltados como motivos de orgullo. Si bien son valores que refuerzan la identidad de las mujeres en sus roles tradicionales, también son expresiones de reconocimiento. El uso estratégico de su identidad como cocineras no sólo les permite abrirse un espacio socialmente legitimado en el mundo del trabajo, sino también interpretar su labor en términos de valía y autoestima.

Más aún, esta capacidad de resignificación también podría estar desigualmente distribuida. Como tendencia general, los negocios más estables y económicamente redituables - los comedores caseros-, permitieron que las madres y cocineras adquirieran mayor autoridad frente a sus hijas, hijos y cónyuges, quienes frecuentemente participaban en los negocios. En estos casos, las narrativas expresan angustia por la precariedad, pero también son un poco más solidarias y destacan el esfuerzo común por la subsistencia familiar. Al hacer explícito el arreglo conyugal al que Irma y Pablo llegaron cuando distribuyeron sus recursos para la construcción de la casa y la subsistencia familiar, Irma reclama su lugar en la historia, el reconocimiento de su esfuerzo. Por su parte, Julia, casi inmediatamente después de haber sancionado públicamente la irresponsabilidad de su marido exalta los resultados del esfuerzo conjunto y reconoce el trabajo realizado:

... gracias a dios, en esta casa no hubo electricista... Aquí el plomero, el albañil, el pegazulejo, fue él. Yo no sé de dónde sacó todo, pero él lo hizo... No puedo decir que no, a la hora que fuera, en la noche... O sea, esta casa está hecha con muchos esfuerzos por mi propia familia.

Rebeca, por su parte, recuerda haber hecho famosa a su fonda por el mole "amarillito" que preparaba, sus frijoles rancheros y el servicio que brindaban. A Julia le enorgullece la osadía con la que un día decidió vender comida por su cuenta. "Yo me aventé sola, sin medidas ni nada; a mí nada más me decían 'es para tanta gente' y órale". Destaca que además "se dio a querer", se hizo popular, obtuvo estima y preferencia. Los testimonios expresan orgullo por el trabajo duro, por la constancia e incluso por la fatiga y los sacrificios. Así le ocurría a Ana en las cocinas industriales donde trabajó: "Sí eran chingas, la verdad. Había que darle de comer a los empleados, vete a bufet, sácame los pozoles, guarniciones, refríeme dos bolsas de frijoles. Entonces, imagínate...”. Sin embargo, su trabajo le gustaba, se sentía competente, llegando incluso a desafiar la autoridad de sus superiores. Ana modificaba los platillos que ya venían preparados si los encontraba insípidos; les agregaba sal, especias, más carne. En su relato, los chefs intentaban detenerla, pero ella se 
negaba a obedecer: “a mí no me va a decir cómo hacerlo, yo lo voy a hacer como yo sé porque así queda bueno".

Por su parte, con menos recursos y más problemas económicos, Maricela y Beatriz también valoran haberse atrevido a vender alimentos y estar dispuestas a hacer lo necesario por su familia —un sacrificio que representa la quintaesencia de la maternidad-, pero en sus casos los bajos rendimientos y lo complicado que resultaba mantener sus negocios modelaron una narrativa distinta que no es tan generosa consigo mismas, como la de sus pares con actividades más prósperas y duraderas. Estas mujeres con trabajos inestables y peor pagados, expresaron mayor cansancio, desaliento y soledad en sus empeños, así como poco o nulo apoyo en el trabajo del hogar. Y es que, a pesar de que las narrativas de las mujeres hablan de compromiso por el bienestar familiar, su disposición solidaria no encontró correspondencia masculina en el ámbito del trabajo doméstico y de cuidados.

Tenemos entonces que la incorporación subjetiva de la división sexual del trabajo y de sus desigualdades funciona de manera dual: por un lado, normaliza el menosprecio por el trabajo doméstico y de cuidados y extiende su desvalor social y económico al mercado laboral. Por otro, la división sexual del trabajo, en tanto dispositivo ideológico, tiene la peculiaridad de afirmar habilidades específicas de lo que se considera ser mujer, entre ellas, saber cocinar, tener buena sazón, ser deferente, esforzada al punto del sacrificio. La percepción del propio desempeño en estos emprendimientos tiene un correlato económico y material que distribuye mayores rendimientos a quienes pudieron emprender negocios más complejos, precisamente porque se encontraban en mejores condiciones, aun en un contexto de precariedad generalizada. Por el contrario, quienes experimentaban mayores limitaciones e iniciaron negocios de menor alcance, recibieron retribuciones económicas y simbólicas menos evidentes.

\section{Discusión: dualidades y desigualdades del trabajo de alimentar}

Las mujeres urbanas de sectores populares se enfrentan a un mercado laboral terciarizado, precarizado, predominantemente informal, poco productivo y con remuneraciones muy bajas. En estas circunstancias, y frente a la dificultad de delegar el trabajo reproductivo en alguien más - 
familiar, privado o institucional—, las mujeres realizan actividades que permitan empalmar el trabajo doméstico con el extradoméstico. Esta dinámica dificulta pensar en los espacios públicos y privados como dos entes distintos. Insistir en ello no sólo refuerza la separación impuesta por la división sexual del trabajo, sino que oscurece las prácticas que conectan cotidianamente los dos ámbitos a través de procesos de mutua configuración entre el espacio y las relaciones de género (Gal, 2002; Rose, 2007; Zelizer, 2005).

Las mujeres que se dedican a la venta de alimentos desde y para estratos populares, en negocios situados dentro de sus propios territorios, articulan lo privado con lo público en la convergencia entre el trabajo alimentario para la familia y para el comercio. Para ello las mujeres hacen uso de los recursos que les han sido asignados mediante su socialización como mujeres, madres y cuidadoras, así como de sus relaciones familiares, sus posesiones materiales, su tiempo y su propia fuerza de trabajo. El hecho de que la actividad económica que realizan sea una extensión del trabajo doméstico implica que ambas esferas compartan una ideología sobre el valor social y económico de su trabajo, lo que se traduce en la reproducción de desigualdades que sobrecargan a las mujeres al mismo tiempo que escatiman la importancia de sus aportes. Es decir, si como señala DeVault (1991) el trabajo de alimentar refleja una idea de familia y de la posición femenina permanentemente orientada hacia otros, la venta del trabajo de alimentar extiende ese imaginario al mercado.

A pesar de ello, la participación laboral de las mujeres en la economía informal, frecuentemente motivada por la posición desventajosa de género y clase en la que se encuentran, también puede ser experimentada como una fuente de orgullo o reconocimiento. En el caso de la venta del trabajo alimentario se aprecia el valor de las habilidades culinarias, la eficiencia en la realización de las tareas o la deferencia en el servicio. La dualidad entre la desvalorización y el reconocimiento en la experiencia del trabajo femenino se expresa en: las motivaciones para iniciar estos negocios (constreñimientos económicos o expresiones de dominación masculina que, a pesar de sus formas opresivas, provocan la iniciativa de las mujeres que movilizan su agencia con los recursos disponibles); los arreglos entre el trabajo público y el doméstico (que llevan a las mujeres a asumir sobrecargas de trabajo que, sin embargo pueden mejorar su posición de poder en la familia); las retribuciones económicas y simbólicas (ganancias variables que no necesariamente corresponden 
con el esfuerzo desplegado, pero aportan reconocimiento a la sazón, orgullo por la preferencia de los comensales y fortalecimiento de redes de intercambio).

Así, cuando los cuidados y el trabajo de alimentar son experimentados como un trabajo experto y significativo pueden ser fuente de afirmación. Por el contrario, cuando son vividos estrictamente como servicios personales poco satisfactorios pueden llevar a las mujeres hacia el autosacrificio o la frustración (Oakley, 2019). Si bien no es el único factor, la pobreza puede hacer la diferencia entre uno y otro escenario. Contar con recursos económicos, materiales y simbólicos permite mantener estilos de vida que se consideran legítimos y deseables, afectando positivamente la calidad de las dietas, la percepción de las mujeres sobre su trabajo y la atención que ponen a sus propias preferencias e intereses alimentarios. Por el contrario, la falta de recursos se traduce en dietas deficientes y menos satisfactorias, así como mayor ansiedad y culpa en torno al desempeño maternal (Charles y Kerr, 1986b; Cairns y Johnston, 2016; Bowen, Brenton y Elliot, 2019; O’Conell y Brannen, 2016; Villagómez, 2019).

El acervo de recursos económicos y materiales también puede inclinar positiva o negativamente el balance del rol alimentario femenino, tanto en lo familiar como en lo laboral. Las disparidades observadas incluso dentro de un mismo territorio- acentúan la experiencia negativa del rol para quienes tienen menos dinero para emprender negocios más redituables y duraderos, mientras que a mayores recursos corresponden experiencias más positivas, no sólo económicas sino también simbólicas. Estos resultados, sin embargo, no se presentan como escenarios excluyentes; las experiencias no son "buenas" o "malas", sino que ambos saldos económicos, morales y emocionales coexisten e incluso pueden derivarse de una misma situación, por ejemplo, al sentirse abrumada por la sobrecarga de trabajo y el desdén de las parejas y, al mismo tiempo, disfrutar los procesos de la cocina y la convivencia con los comensales.

\section{Comentarios finales}

La cocina es un epicentro de negociación entre las necesidades de las mujeres y los mandatos sociales; es un espacio de la socialización que no está permanentemente definido y no siempre opera de la misma manera, sino que acepta cambios y reposicionamientos. En este sentido, vale la 
pena pensar en los discursos que expresan satisfacción con este tipo de actividades no sólo como un orden interiorizado, sino también como una forma de agencia producida desde la reflexividad que puede tener resultados positivos en la percepción de las mujeres sobre sí mismas. Se trata de pensar en la identidad y sus roles como un recurso estratégico complejo que se construye en diálogo

con otros y que, dependiendo de las circunstancias, puede enfatizar aspectos opresivos o constructivos.

Ante la dificultad que experimentan muchas mujeres para evadir el trabajo de alimentar, es necesario analizar cómo despliegan su agencia para resignificar este trabajo a través de la construcción de diversas maneras de autorepresentarse. La apropiación de las cocinas como espacios transformadores no niega la historia opresiva que les ha dado forma, pero no parece justo omitir la voz de las mujeres que encuentran un sentido positivo en asumirse como cocineras y obtienen recompensas económicas o simbólicas de su trabajo. Sin embargo, tampoco es conveniente que alimentar sea reconocido como un trabajo sólo cuando se enmarca en la lógica del mercado.

Lo deseable es que se revierta la doble exclusión que representa para las mujeres que el trabajo reproductivo no sea reconocido como tal y que, al mismo tiempo, lo que sí lo constituye - una actividad económica remunerada en el sector formal - sea el único mecanismo de acceso a sistemas de protección social. En el caso del trabajo alimentario informal, valorar con justicia su aportación al circuito de subsistencia y a la seguridad alimentaria también significa replantear el imaginario del espacio público, un sitio por el que muchas mujeres trabajadoras aún transitan como invitadas de segunda categoría.

\section{Agradecimientos}

La autora agradece la lectura y los comentarios de las personas dictaminadoras. Este texto fue elaborado con el apoyo del Programa de Becas Posdoctorales en la Universidad Nacional Autónoma de México (UNAM), donde la autora es becaria del Instituto de Investigaciones Sociales y es asesorada por la Dra. Fiorella Mancini. 


\section{Referencias bibliográficas}

Abarca, Meredith. (2006). Voices in the Kitchen. Views of Food and the World from Working-Class Mexican and Mexican American Women. Estados Unidos: Texas A\&M University Press.

Abarca, Meredith. (2007). Charlas culinarias: Mexican Women Speak from their Public Kitchens. Food \& Foodways, 15(3-4), 183-212. doi: https://doi.org/10.1080/07409710701620094

Ariza, Marina. (2006). Mercados de trabajo y desigualdad de género en México a principios del siglo XXI. En Enrique de la Garza y Carlos Salas (Coords.), La Situación del Trabajo en México (pp. 377-411). México: Universidad Autónoma Metropolitana.

Arriagada, Irma. (2005). Los límites del uso del tiempo: dificultades para las políticas de conciliación, familia y trabajo. En Irma Arriagada (Ed.), Políticas hacia las familias, protección e inclusión sociales (pp. 131-148). Santiago: CEPAL, División de Desarrollo Social.

Avakian, Arlene y Haber, Barbara. (2005). Feminist food studies. A brief history. En Arlene Avakian y Barbara Haber (Eds.), From Betty Crocker to Feminist Food Studies. Critical perspectives on women and food (pp. 1-26). Boston: University of Massachusetts Press.

de Barbieri, Teresita. (1978). Notas para el estudio del trabajo de las mujeres: El problema del trabajo doméstico. Demografia y Economía, XII(1), 129-137. doi: http://dx.doi.org/10.24201/edu.v12i01.421

Beagan, Brenda; Chapman, Gwen y Power, Elain. (2017). The visible and invisible occupations of food provisioning in low income families, Journal of Occupational Science, 25(1), 1-13. doi: https://doi.org/10.1080/14427591.2017.1338192 
Beagan, Brenda; Chapman, Gwen y Power, Elaine. (2018). Cultural and Symbolic Capital With and Without Economic Constraint: Food Shopping in Low-income and High-income Canadian Families. En Jennifer Smith (Ed.), Food Practices and Social Inequality. Looking at Food Practices and Taste Across the Class Divide (pp. 35-59). Londres: Routledge.

Bowen, Sarah; Brenton, Joslyn y Elliot, Sinikka. (2019). Pressure Cooker. Why Home Cooking Won't Solve our Problems and What Can We Do About It. Reino Unido: Oxford University Press.

Cairns, Kate y Jhonston, Josée. (2016). Food and Femininity. Bloomsbury Academic.

Carney, Megan. (2015). The Unending Hunger. Tracing Women and Food Insecurity Across borders. California: University of California Press.

Cerrutti, Marcela. (2000). Determinantes de la participación intermitente de las mujeres en el mercado de trabajo del Área Metropolitana de Buenos Aires. Desarrollo Económico, 39(156), 619-638. doi: https://doi.org/10.2307/3455835

Charles, Nickie y Kerr, Marion. (1986a). Women, food and families. Manchester: Manchester University Press.

Charles, Nickie y Kerr, Marion. (1986b). Eating properly, the family and the state benefit. Sociology, 20(3), 412-429. Recuperado de https://www.jstor.org/stable/42854300

Charles, Nicola y Kerr, Marion. (1986c). Servers and providers: the distribution of food within the family. The Sociological Review, 34(1), 115-157. doi: https://doi.org/10.1111/j.1467954X.1986.tb02697.x

Cohen, Monique; Bhatt, Mihir y Horn, Pat. (2000). Women Street Vendors: The Road to Recognition. SEEDS, No. 20. Nueva York: The Population Council. 
Cohen, Monique. (1990). Women and the street food trade: Some implications for policy. Gender and Planning Working Paper, No. 50. Recuperado de https://www.ucl.ac.uk/bartlett/development/sites/bartlett/files/migrated-files/WP55 0.pdf

Consejo Nacional de Evaluación de la Política de Desarrollo Social, Coneval. (8 de marzo de 2021a). Medición de la pobreza. Anexo estadístico de la pobreza en México. Recuperado de https://www.coneval.org.mx/Medicion/MP/Paginas/AE_pobreza_2018.aspx

Consejo Nacional de Evaluación de la Política de Desarrollo Social, Coneval. (8 de marzo de 2021b). Medición de la pobreza. Anexo estadístico de pobreza a nivel municipio 2010 y 2015. Recuperado de https://www.coneval.org.mx/Medicion/Paginas/AE_pobreza_municipal.aspx

Consejo Nacional de Evaluación de la Política de Desarrollo Social, Coneval. (8 de marzo de 2021c). Evolución de las líneas de pobreza por ingresos. Recuperado de https://www.coneval.org.mx/Medicion/MP/Paginas/Lineas-de-bienestar-y-canastabasica.aspx

Cross, John. (2000). Street vendors, and postmodernity: conflict and compromise in the global economy. International Journal of Sociology and Social Policy, 20(1-2), 29-51. doi: https://doi.org/10.1108/01443330010789061

Crossa, Veronica. (2016). Reading for difference on the street: De-homogenising street vending in Mexico City. Urban Studies, 53(2), 287-301. doi: https://doi.org/10.1177/0042098014563471

Crossa, Veronica. (2018). Luchando por un espacio en la Ciudad de México: comerciantes ambulantes y el espacio público urbano. Ciudad de México: El Colegio de México, Centro de Estudios Demográficos, Urbano y Ambientales. 
Delgado, Victoria, y Bertran, Miriam. (2010). Consumo de comida callejera y riesgo de obesidad en la ciudad de México. Una aproximación antropológica. En Florencia Peña y Beatriz León (Coords.), La medicina social en México IV. Alimentación cuerpo y corporeidad (pp. 43-53). México: Eón Sociales.

DeVault, Marjorie. (1991). Feeding the family: The social organization of caring as gendered work. Chicago: The University of Chicago Press.

Ehrenreich, Barbara y English, Deirdre. (2005). For her own good. Two centuries of the experts' advice to women. Nueva York: Anchor Books.

Gaceta Oficial de la Ciudad de México. (3 de mayo de 2019). Aviso por el que se dan a conocer las Reglas de Operación del Programa "Comedores Públicos de la Ciudad de México 2019" y Aviso por el que se dan a conocer las Reglas de Operación del Programa "Comedores Comunitarios de la Ciudad de México 2019", No. 13, Tomo 1. Recuperado de https://tubienestarhistorico.cdmx.gob.mx/storage/app/uploads/public/documentos/2019/secr etarias/sibiso/10sibiso_comedorescomuncdmx.pdf

Gal, Susan. (2002). A semiotics of the public/private distinction. A Journal of Feminist Cultural Studies, 13(1), 77-95. Recuperado de: https://www.researchgate.net/publication/30964286_A_Semiotics_of the_PublicPrivate_D $\underline{\text { istinction }}$

García, Brígida y de Oliveira, Orlandina. (2006). Participation in the labor force, women's social position and reproductive behavior: An analysis of the progress achieved to date. En José Luis Lezama y José Morelos (Eds.), Population, City and Environment in Contemporary Mexico, (pp. 203-222). México: El Colegio de México.

García, Brígida y de Oliveira, Orlandina. (2004). Trabajo extradoméstico femenino y relaciones de género: una nueva mirada. Estudios Demográficos y Urbanos, 19(1), 145-180. doi: http://dx.doi.org/10.24201/edu.v19i1.1198 
González de la Rocha, Mercedes. (1994). The Resources of Poverty. Women and Survival in a Mexican City. Oxford: Basil Blackwell Publishers.

Hayden, Tiana Bakic. (2014). The taste of precarity. Language, legitimacy, and legality among Mexican street food vendors. En Ryzia De Cassia Vieira Cardoso, Michèle Companion, Stefano Roberto Marras (Eds.), Street Food: Culture, Economy, Health and Governance (pp. 83-97). Londres: Routledge.

Hayden, Tiana Bakic. (2018). Disambiguating legalities: Street vending, law, and boundary-work in Mexico City. EthnoScripts, The Anthropology of Work and Labour, 19(2), 14-30. Recuperado de https://journals.sub.uni-hamburg.de/ethnoscripts/article/view/1174

Instituto Nacional de Estadística y Geografía, INEGI. (2019a). Resultados de la Encuesta Nacional de Ocupación y Empleo. Cifras correspondientes al tercer trimestre de 2019. Comunicado $\begin{array}{llll}\text { de } & \text { prensa } & \text { No. } & \text { R55/19. }\end{array}$ https://www.inegi.org.mx/contenidos/saladeprensa/boletines/2019/enoe ie/enoe ie2019 11 . $\mathrm{pdf}$

Instituto Nacional de Estadística y Geografía, INEGI. (2019b). Producto Interno Bruto de México durante el tercer trimestre de 2020. Comunicado de prensa NO. 606/20. Recuperado de https://www.inegi.org.mx/contenidos/saladeprensa/boletines/2020/pib_pconst/pib_pconst20 $\underline{20 \_11 . p d f}$

Julier, Alice. (2005). Hiding gender and race in the discourse of commercial food consumption. En Arlene Avakian y Barbara Haber (Eds.), From Betty Crocker to Feminist Food Studies. Critical Perspectives on Women and Food (pp. 163-184). Boston: University of Massachusetts.

Kabeer, Naila. (2010). Gender and social protection strategies in the informal economy. Londres: Routledge. 
Khurana, Sakshi. (2015). Negotiating responsibilities and relationships: power, conflict, and decision-making in households of informal women working. Asian Journal of Women's Studies, 21(3), 273-294. doi: https://doi.org/10.1080/12259276.2015.1072942

McLean, Alice. (2013). The intersection of gender and food studies. En Ken Albala (Ed.), Routledge International Handbook of Food Studies (pp. 250-264). Nueva York: Routledge.

Mennell, Stephen; Murcott Anne y Van Otterloo, Anneke H. (1992). The Sociology of Food: Eating, Diet and Culture. Londres: Sage.

Murcott, Anne. (1993). Talking of good food: An empirical study of women's conceptualizations. Food and Foodways, 5(3), 305-318. doi: https://doi.org/10.1080/07409710.1993.9962010

O’Connel, Rebecca y Brannen, Julia. (2016). Food, families and work. Reino Unido: Bloomsbury.

Oakley, Ann. ([1974] 2019). The Sociology of Housework. Reino Unido: Policy Press.

Oleschuk, Merin. (2019). Gender, Cultural Schemas and Learning to Cook. Gender \& Society, $X X(\mathrm{X}), 1-22$. doi: https://doi.org/10.1177/0891243219839669

de Oliveira, Orlandina y Ariza, Marina. (2000). Género, trabajo y exclusión social en México. Estudios Demográficos y Urbanos, 15(1), 11-33. doi: http://dx.doi.org/10.24201/edu.v15i1.1065

de Oliveira, Orlandina; Eternod, Marcela y López, Paz. (1999). Familia y género en el análisis demográfico. En Brígida García (Coord.), Mujer, género y población en México (pp. 211 271). México: El Colegio de México, Sociedad Mexicana de Demografía.

Organización de las Naciones Unidas para la Agricultura y la Alimentación, FAO. (2007). Promesas $y$ desafios del sector alimentario informal en países en desarrollo. Roma: FAO. Recuperado de http://www.fao.org/sustainable-food-value-chains/library/detalles/es/c/278595/ 
Parsons, Julie. (2015). Gender, class and food. Families, body and health. Inglaterra: McMillan.

Pérez Sáinz, Juan Pablo. (1999). Mercado laboral, integración social y modernización globalizada en Centroamérica. Nueva Sociedad, (164), 106-121. Recuperado de https://nuso.org/articulo/mercado-laboral-integracion-social-y-modernizacion-globalizadaen-centro-america/

Pérez, Juan Pablo y Mora, Minor. (2006). Exclusión social, desigualdades y excedente laboral. Reflexiones analíticas sobre América Latina. Revista Mexicana de Sociología, 68(3), 431465. doi: http://dx.doi.org/10.22201/iis.01882503p.2006.003.6066

Rodríguez, Corina. (2015). Economía feminista y economía del cuidado. Aportes conceptuales para el estudio de la desigualdad. Nueva Sociedad, (256). Recuperado de: https://nuso.org/articulo/economia-feminista-y-economia-del-cuidado-aportesconceptuales-para-el-estudio-de-la-desigualdad/

Rose, Gillian. (2007). Feminism and Geography. The limits of geographical knowledge. Cambridge: Polity Press.

Scott, Joan. (1990). El género: una categoría útil para el análisis histórico. En Mary Nash y James Amelang (Eds.), Historia y género: las mujeres de la Europa moderna y contemporánea (pp. 23-58) Valencia: Alfons el magnanim.

Tinker, Irene. (1997). Street Foods. Urban Food and Employment in Developing Countries. Nueva York: Oxford University Press.

Tinker, Irene. (2003). Street Foods: Traditional Microenterprise in a Modernizing World. International Journal of Politics, Culture and Society, 16(3), 331-349. Recuperado de https://ink.springer.com/article/10.1023/A:1022300210762 
Villagómez, Paloma. (2019). La Constitución Social de Experiencias Alimentarias en la Pobreza: Un estudio cualitativo con familias de estratos populares en Iztapalapa, Ciudad de México. (Tesis de doctorado en Ciencia Social con especialidad en Sociología). El Colegio de México, Ciudad de México. $\quad$ Recuperada de https://repositorio.colmex.mx/concern/theses/br86b401b?locale=es

West, Candance y Fenstermaker, Sarah. (1995). Doing difference. Gender \& Society, 9(1), 8-37. Recuperado de https://www.jstor.org/stable/189596

Women in Informal Employment: Globalizing and Organizing, WIEGO. (15 de mayo de 2019). Alimentar a las ciudades: el comercio informal juega un papel clave en la seguridad alimentaria de las ciudades. Entrada en el blog de WIEGO. Recuperado de https://www.wiego.org/blog/alimentar-las-ciudades-el-comercio-informal-juega-un-papelcrucial-en-la-seguridad-alimentaria-

Zelizer, Viviana. (2005). The Purchase of Intimacy. Princeton University Press.

\section{Paloma Villagómez ORNElas}

Es Socióloga por la Universidad de Guadalajara, Maestra en Población y Desarrollo por la Facultad Latinoamericana de Ciencias Sociales (FLACSO-México) y Doctora en Ciencia Social con Especialidad en Sociología por El Colegio de México. Cuenta con varios años de experiencia en el sector público y la academia con proyectos relacionados con la pobreza y la desigualdad. Actualmente es investigadora posdoctoral del Instituto de Investigaciones Sociales de la Universidad Nacional Autónoma de México (IISUNAM), donde desarrolla proyectos relacionados con desigualdad social y alimentación. 


\section{Anexo 1. Características de los grupos de hogares según nivel de precariedad socioeconómica}

\section{Cuadro 1. Perfiles de los grupos socioeconómicos identificados}

\begin{tabular}{|c|c|}
\hline $\begin{array}{c}\text { Nivel de } \\
\text { precariedad } \\
\text { [número de } \\
\text { casos] }\end{array}$ & Perfil del grupo \\
\hline \multirow{9}{*}{ Alta [3] } & $\begin{array}{l}\text { - Ingresos corrientes totales per cápita menores al costo de la canasta básica } \\
\text { alimentaria. }\end{array}$ \\
\hline & - Fase de formación o expansión del ciclo doméstico. \\
\hline & - Familias más numerosas (5 miembros en promedio). \\
\hline & $\begin{array}{l}\text { - Relaciones de dependencia económica más tensas, dada la presencia de más } \\
\text { consumidores y menos proveedores. }\end{array}$ \\
\hline & $\begin{array}{l}\text { - Baja o nula participación laboral femenina; predominancia de jefaturas económicas } \\
\text { masculinas. }\end{array}$ \\
\hline & - Viviendas precarias, frecuentemente rentadas o prestadas. \\
\hline & - Falta de equipamiento y servicios básicos en las viviendas. \\
\hline & - Ubicación de mayor aislamiento geográfico dentro de las colonias. \\
\hline & - Mayor presencia de enfermedades. \\
\hline \multirow{8}{*}{ Media [4] } & $\begin{array}{l}\text { - Ingresos corrientes totales per cápita por debajo del costo de la canasta alimentaria } \\
\text { y no alimentaria. }\end{array}$ \\
\hline & - Mayor presencia de hogares en fase de dispersión del ciclo doméstico. \\
\hline & - Mayor presencia de estructuras familiares extensas. \\
\hline & - Familias de 4 miembros en promedio. \\
\hline & - Mayor participación laboral femenina. \\
\hline & $\begin{array}{l}\text { - Relaciones de dependencia económica menos tensas por la presencia de más } \\
\text { proveedores económicos. }\end{array}$ \\
\hline & $\begin{array}{l}\text { - Viviendas consolidadas en términos de construcción, pero con carencia de equipos } \\
\text { y servicios deficientes. }\end{array}$ \\
\hline & - Residencia en áreas mejor comunicadas de las colonias. \\
\hline \multirow{8}{*}{ Baja [6] } & $\begin{array}{l}\text { - Ingresos por encima del costo de la canasta de necesidades básicas alimentarias y } \\
\text { no alimentarias. }\end{array}$ \\
\hline & - Hasta tres miembros familiares en promedio. \\
\hline & - Hogares en diferentes fases del ciclo doméstico. \\
\hline & $\begin{array}{l}\text { - Relaciones de dependencia económica más holgadas, dado el menor tamaño } \\
\text { familiar. }\end{array}$ \\
\hline & - Jefaturas económicas masculinas. \\
\hline & - Trayectorias laborales asociadas a trabajos con seguridad social. \\
\hline & $\begin{array}{l}\text { - Viviendas consolidadas, con equipamiento suficiente y adecuado, aunque con } \\
\text { servicios deficientes. }\end{array}$ \\
\hline & - Residencia en áreas mejor comunicadas de las colonias. \\
\hline
\end{tabular}

